\title{
Anti-inflammatory Activity of Curcumin in Gel Carriers on Mice with Atrial Edema
}

\author{
Luis A. González-Ortega1, Andrés A. Acosta-Osorio ${ }^{4 *}$, Peter Grube-Pagola², \\ Carolina Palmeros-Exsome ${ }^{3}$, Cynthia Cano-Sarmiento ${ }^{4}$, Rebeca García-Varela ${ }^{5,6}$, and \\ Hugo S. García ${ }^{1 *}$ \\ ${ }^{1}$ UNIDA, Tecnológico Nacional de México/IT de Veracruz. Miguel Ángel de Quevedo 2779. Veracruz, Ver. MÉXICO. 91897 \\ ${ }^{2}$ Universidad Veracruzana, Instituto de Investigaciones Médico Biológicas, Iturbide s/n, Veracruz, Ver. 91700, MÉXICO \\ ${ }^{3}$ Universidad Veracruzana, Facultad de Nutrición, Iturbide s/n, Veracruz, Ver. 91700, MÉXICO \\ ${ }^{4}$ CONACyT-Tecnológico Nacional de México/IT de Veracruz. Miguel Ángel de Quevedo 2779. Veracruz, Ver. MÉXICO. 91897 \\ ${ }^{5}$ Department of Medicine, Hematology/Oncology, UW Carbone Cancer Center, University of Wisconsin at Madison, School of Medicine and \\ Public Health, Madison, WI 53705, USA \\ ${ }^{6}$ Tecnologico de Monterrey, Escuela de Ingenieria y Ciencias, Av. General Ramón Corona 2514, Nuevo México 45138, Zapopan, Jalisco, \\ MÉXICO
}

\begin{abstract}
Curcumin is a bioactive compound with proven antioxidant and anti-inflammatory activities, but has low water solubility and dermal absorption. The inflammatory process is considered as the biological response to damage induced by various stimuli. If this process fails to self-regulate, it becomes a potential risk of cancer. The objective of this work was to evaluate the anti-inflammatory activity of curcumin administered to mice with induced atrial edema using two topical vehicles: organogels and $O / W$-type nanogels at pH 7, Organogels and $\mathrm{O} / \mathrm{W}$-type nanogels at pH 7 were prepared, characterized and the antiinflammatory activity was assessed. A histopathological analysis of mouse ears was performed and two gel formulations were selected. Thermograms of organogels indicated that increasing the gelling agent improved the stability of the system. Deformation sweeps confirmed a viscoelastic behavior characteristic of gels in both systems. During the anti-inflammatory activity evaluations, the nanogels demonstrated greater activity (61.8\%) than organogels; Diclofenac ${ }^{\circledR}$ (2-(2,6-dichloranilino) phenylacetic acid), used as a control medication achieved the highest inhibition (85.4\%); however, the drug produced the death of $2(40 \%)$ of the study subjects caused by secondary adverse events. Histopathological analysis confirmed the data.
\end{abstract}

Key words: curcumin, nanogel, organogel, bioactivity, anti-inflammatory

\section{Introduction}

Inflammation is defined as a biological response to damage induced by certain stimuli, an essential mechanism for the body to maintain a homeostatic state. Although it is normally regulated, in some cases the process fails to function adequately leading to acute inflammation and becomes a potential risk to the organism. When an inflammatory response persists, a chronic inflammation diagnosis can be established, which may function as a trigger for several chronic pathologies ${ }^{1)}$. The consumption and application of medicinal plants as treatments against regular or chronic inflammation has been widely used since ancient times, with reported beneficial effects. It is now known that specific bioactive compounds are responsible for the attributed health enhancing properties of these plants ${ }^{2,3)}$.
A bioactive compound is a product that provides health benefits when consumed, that may include anti-inflammatory, antioxidant activity, ROS and RNS scavenging, specific cytotoxicity, among others. These properties may aid in the prevention and/or mitigation of some chronic diseases ${ }^{4}$. Curcumin, known as the "Indian gold", has been used to alleviate arrhythmia, and as an aid in the treatment of various illnesses including Alzheimer's, asthma, liver disease, and also as antioxidant, anti-inflammatory, cardioprotective, anticarcinogenic and chemopreventive agent ${ }^{5-7)}$. Curcumin has been reported to interfere in several mechanisms that control inflammatory responses, among which are: modulation of arachidonic acid metabolism ${ }^{8)}$, inhibition of cyclooxygenase 2 (COX-2) and lipoxygenase (LOX), two enzymes involved in inflammation. COX-2 induces cyto-

\footnotetext{
*Correspondence to: Hugo S. García, UNIDA, Tecnológico Nacional de México/IT de Veracruz. Miguel Ángel de Quevedo 2779. Veracruz, Ver. MÉXICO. 91897; Andres A. Acosta-Osorio, CONACyT-Tecnológico Nacional de México/IT de Veracruz. Miguel Ángel de Quevedo 2779. Veracruz, Ver. MÉXICO. 91897.

E-mail: hugo.gg@veracruz.tecnm.mx (HSG), andres.ao@veracruz.tecnm.mx (AAAO)

Accepted December 3, 2019 (received for review August 24, 2019)
}

Journal of Oleo Science ISSN 1345-8957 print / ISSN 1347-3352 online

http://www.jstage.jst.go.jp/browse/jos/ http://mc.manusriptcentral.com/jjocs 
kines that transform arachidonic acid in prostaglandins and thromboxanes during episodes of inflammation and platelet aggregation; whereas LOX transforms arachidonic acid into leukotrienes, which are involved in the recruitment of leukocytes by endothelial cells and their mobilization from the vasculature to damaged tissues ${ }^{6)}$. Curcumin is also able to inhibit COX activity by suppressing the activation of $\mathrm{NF}-\kappa \mathrm{B}$ factor; this factor regulates the expression of COX-2 and inhibits the expression of the pro-inflammatory enzyme LOX-5, inflammatory cytokines, chemokines, nitric oxide synthase, and reduces the production of pro-inflammatory cytokines such as tumor necrosis factor alpha $(\mathrm{TNF} \alpha)^{9,10)}$. This cytokine regulates the expression of pro-inflammatory genes $^{11)}$, and the expression of several inflammatory cytokines such as IL-1, and IL6, involved in inflammatory responses and further progression towards cancer ${ }^{12)}$. It is also a potent inhibitor of the production of free oxygen radicals, thereby reducing inflammation ${ }^{13)}$. Despite all the previously reported beneficial properties, curcumin has as a major drawback its low solubility in aqueous media, of only $0.6 \mu \mathrm{g} / \mathrm{mL}$. When $10-12 \mathrm{~g} / \mathrm{mL}$ were orally administered to humans, curcumin serum levels did not exceed $50 \mathrm{ng} /$ $\mathrm{mL}$. This amount was below the suggested necessary concentration required to achieve significant therapeutic effects $^{14)}$.

On the other hand, curcumin has a rapid biological degradation rate and is susceptible to breakdown in alkaline media. These traits characterize curcumin as a bioactive compound with low bioavailability, resulting in a suboptimal concentration in blood, not sufficient for reaching health-enhancing effects ${ }^{15)}$. Because of this limitation, the design of carriers capable of increasing its concentration in the body, solving the solubility aspect and protect this hydrophobic material, have been pursued ${ }^{16,17)}$. A gel system is highly biocompatible and biodegradable, and thus accumulation in the body is prevented. The gel preparation process is relatively easy since it does not require toxic reagents or catalysts nor generate byproducts, which makes them appropriate and favorable for biomedical applications.

\section{Chemicals and Reagents}

\subsection{Materials and reagents}

All chemical compounds and solvents used were analytical or HPLC grade; soy lecithin (98\% purity, Shenyang, Liaoning, China) was select as emulsifier. For nanoemulsions, curcumin (98\% purity, LKT Laboratories, St. Paul, MN) was used; reactive grade glycerol(JT Baker, Mexico City). For gel formulations, nanogels required Carbopol $940^{\circledR}$ (Polyacrylic acid) (Lubrizol, Mexico City), whereas Phospholipon ${ }^{\circledR} 90 \mathrm{H}$ (Phosphatidylcholine, hydrogenated), Lipoid, Ludwigshafen, Germany) was employed for organo- gels as gelling agents; Diclofenac ${ }^{\circledR}$ (2-(2,6-dichloranilino) phenylacetic acid) was used as an anti-inflammatory drug. 12-O-tetradecanoylphorbol 13-acetate (TPA, Sigma-Aldrich, Mexico City) was employed to induce a localized inflammation on the ear.

\subsection{Methods}

2.2.1 Formulation of Curcumin nanoemulsions (CNEs)

CNEs were prepared using a dispersed:dispersant phase proportion of 5:95. The dispersed phase was prepared as follows: $25 \mathrm{mg} / \mathrm{g} \mathrm{NE}$ of curcumin, $1 \mathrm{~mL}$ ethanol and $5 \%$ of commercial medium chain triglycerides (MCT, Original Thin $\mathrm{Oil}^{\circledR}$, Sound Nutrition, Dover, ID); this phase was placed in an orbital shaker (Thermo Scientific MaxQ 4450) for 30 minutes at $300 \mathrm{rpm}$ and $45^{\circ} \mathrm{C}$. The dispersant phase consisted of $25 \%$ glycerol, $5 \%$ phosphatidylcholine (PC) and water, manually mixed for 5 minutes. Both phases were sonicated in an Aquawave 9376 (Barnstead/Labline) bath for one minute and incorporated to homogenize for 3 minutes at 20,000 rpm in a T25 digital ULTRA-TURRAX homogenizer (IKA Works, Inc., Wilmington NC), producing a coarse emulsion. Immediately, ultrasonic emulsification was performed at 20\% amplitude, using a Branson Digital S-450D sonicator (Emerson Electric Co., St. Louis MO), fitted with a $13 \mathrm{~mm}$ diameter probe for 3 minutes to obtain the nanometric droplets.

Droplet size was measured in a Nano-Zs90 dynamic light scattering device (Malvern Instruments Inc. Worcestershire, UK) with a $90^{\circ}$ fixed angle at $25^{\circ} \mathrm{C}$; samples were diluted at a rate of 1:200 with deionized water to avoid multiple scattering effects.

\subsubsection{Formulation of Curcumin organogels (COGs)}

To prepare organogels, Phospholipon 90, and curcumin were mixed with MCT in a $1.5 \mathrm{~cm}$ diameter tube. The samples were prepared by dissolving all components using a drying oven at $110^{\circ} \mathrm{C}$, until completely fused. Then, the homogeneous mixture was stirred using a vortex and returned to the stove to melt and achieve complete homogenization; it was then set to cool at room temperature $\left(25^{\circ} \mathrm{C}\right)$ and finally stored in a cooling chamber at $15^{\circ} \mathrm{C}^{18)}$. 2.2.3 Formulation of Curcumin nanogels (CNGs) at $\mathrm{pH} 7$

In order to obtain formulated O/W- nanogels, Carbopol $940^{\circledR}$ was dispersed at a concentration of $0.5,1$ and $1.5 \%$, and set to stand for 6, 12 and 24 hours, for a gel to develop $^{19,20)}$. The resulting mixture was stirred at room temperature and neutralized with triethanolamine to attain a nanogel with a $\mathrm{pH}=7^{21}$.

\subsubsection{Assessment of gel formation}

The inverted tube method is the most commonly used to confirm gel formation. In this method, the gel was placed in a test tube; which was then carefully heated to $110^{\circ} \mathrm{C}$ for a complete melt. The tube was set to cool for 3 hours in a chamber set at $15^{\circ} \mathrm{C}$ and then inverted for one hour at room temperature ${ }^{22,23)}$. After the inversion, the absence of 
flow indicated the formation of a gel, assuming that the sample formed the cross-linked network that prevents the flow of liquid trapped in the matrix ${ }^{24)}$. The nanogels were also analyzed using this test.

\subsubsection{Rheological Characterization}

The rheology of the organogels was analyzed using a Discovery HR2 rheometer(TA Instruments, New Castle, $\mathrm{DE})$ with a cone:plate geometry of $40 \mathrm{~mm}$ in diameter, and a $2^{\circ}$ cone angle, equipped with a Peltier temperature control. To obtain the rheological measurements, the initial position of the orifice was set at $500 \mu \mathrm{m}$. However, according to the thickness of each organogel, the width of the gap was varied in order to use a normal force of zero during the measurements. The deformation sweep was carried out between 0.1 and $1000 \%$, at $25^{\circ} \mathrm{C}$ and a fixed frequency of 1 $\mathrm{Hz}$. Three stages were established: first, heating from $25^{\circ} \mathrm{C}$ to $110^{\circ} \mathrm{C}$ at a rate of $5^{\circ} \mathrm{C} / \mathrm{min}$; second, cooling from $110^{\circ} \mathrm{C}$ to $15^{\circ} \mathrm{C}$, at the same rate; and, third, again from $15^{\circ} \mathrm{C}$ to $110^{\circ} \mathrm{C}$. The formed nanogels were evaluated using the described methodology with some modifications; no changes in temperature were made.

\subsubsection{Bioactivity of curcumin NGs}

For in vivo studies, 35 mice (strain cd1) of 8 weeks of age (22-25 g) were kept under constant temperature (25 \pm $0.5^{\circ} \mathrm{C}$ ), $60 \%$ humidity, $12 / 12$ light/dark periods and ad libitum fodder (Harlan Teklad Global 18\% protein rodent diet 2018S) and water. Animal maintenance and handling were performed according to the NOM-062-ZOO-1999 and the NRC Guide for the Care and Use of Laboratory Animals ( $8^{\text {th }}$ Ed., 2011). Seven groups were randomly formed $(n=$ 5) : group (A) as a healthy control; group (B) only TPA was applied as an edema control; group $(\mathrm{C})$ administered with Diclofenac ${ }^{\circledR}$; group (D) free curcumin in ethanol (FC) (10 $\mathrm{mL})$; group (E) administered with CNGs; $\operatorname{group}(\mathrm{F})$ administered with COGs; group (G) administered with CHGs.

All test groups, except A, were subjected to an ear edema protocol in accordance to Stanley ${ }^{25)}$, using multiple topical applications of TPA; maximum ear thickness was reached on the fifth day. The FC, COGs, CHGs and CNGs groups received the corresponding treatments in each TPA topic application. Mice were euthanized by dorsal dislocation six hours after the last treatment application.

\subsubsection{Histological analysis}

After euthanasia, dissection of swollen ear samples was performed by means of a punch; samples were preserved in $10 \%$ formaldehyde in preparation for histological analysis of all groups. A macroscopic description was made and three slices were taken from each sample, the slices were encapsulated in histocassetes and immersed in 10\% formaldehyde. Subsequently, an automatic tissue processor was employed following a standard protocol that uses ethanol at different concentrations (70\% 80\% 90\% and 100\%); to achieve cell detachment; samples were rinsed with xylol.

Samples were submitted to inclusion by placing in a bath of liquid paraffin at $60^{\circ} \mathrm{C}$; tissue was removed from the histocassete and deposited on a mold were liquid paraffin was added; samples were fixed to the mold using manual pressure; additional paraffin was added and then placed on a surface at $4^{\circ} \mathrm{C}$ to remove the paraffin and proceed to histological cut in a microtome. Once the desired cuts were obtained, samples were observed in an Olympus BX51 microscope with an attached camera using the $10 \mathrm{x}$ and $40 \mathrm{x}$ lenses; cuts were examined in duplicate.

\subsection{Statistical analysis}

Data from the experimental designs were analyzed by means of ANOVA and comparison of means by Tukey's test, to identify pairwise differences between the different treatments, with differences indicated by different superscript letters, with a level of significance of $p<0.05$ using the MINITAB statistical software v17 (Minitab, Inc. College Station, PA).

\section{Results and Discussion}

\subsection{Development of COGs and inverted tube test}

Developed COGs displayed positive results during the inverted tube test (Fig. 1A). Gel formation is caused by micellar level transitions to a low viscosity Newtonian liquid consisting of inverse lecithin micelles in the oil. A solid phase forms a rigid network interconnected with sub-micron size pores that trap and immobilize a liquid phase and resulted in the formation of a three-dimensional network. This is achieved by the presence of small amounts of water in non-aqueous solutions with soy lecithin, which causes a sharp increase in viscosity ${ }^{26)}$.

\subsection{Rheology of COGs}

Table 1 depicts results obtained for the logarithm of G' (storage modulus), expressed in Pascals, as a function of the activation time and the concentration of Phospholipon $90 \mathrm{H}$, where G' had higher values at high concentrations of the gelling agent. When comparing the different formulations, it was determined that $25 \%$ of Phospholipon $90 \mathrm{H}$ with $25 \mathrm{mg}$ of curcumin and $25 \%$ of Phospholipon $90 \mathrm{H}$ with $15 \mathrm{mg}$ of curcumin, were the most elastic COGs obtained and were significantly different from the rest.

These findings suggest that even at low concentrations of crosslinker (Phospholipon 90H) a desired system can be obtained, as confirmed in Fig. 1B, where it can be noted that $G^{\prime}$ is over one order of magnitude greater than $G$ ”.

\subsection{Effect of formulation of curcumin NGs on their $\mathrm{pH}$ val- ues}

NGs had a translucent, yellow coloring with smooth appearance; depending on the amount of polymer present; higher amounts of polymer resulted in less softness and 
A

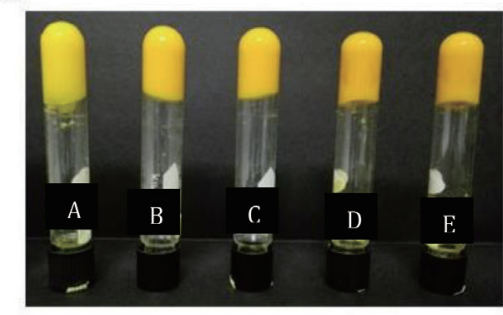

C

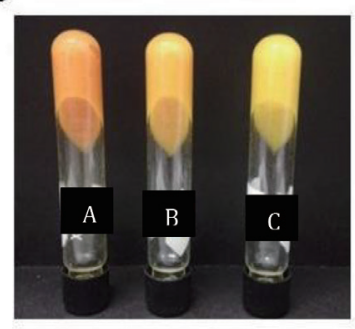

B
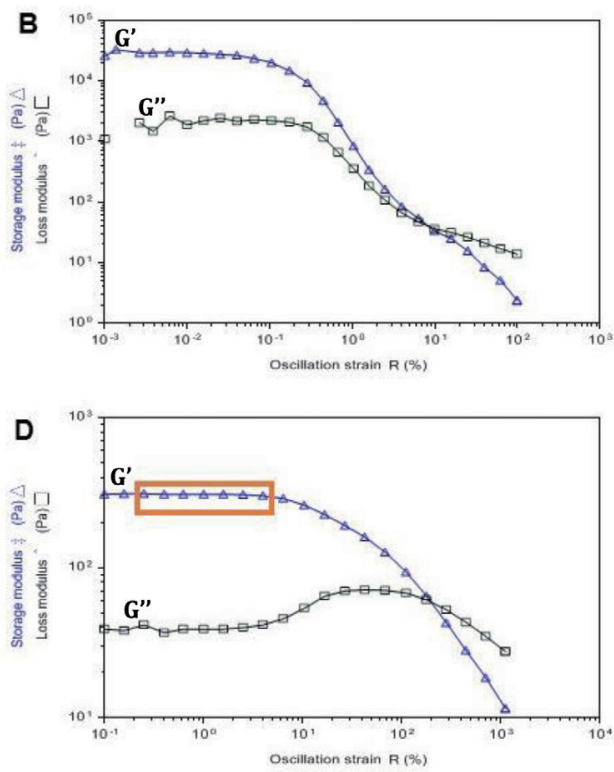

Fig. 1 (A) Organogels developed during the inverted tube test according to the experimental design \%phospholipon 90H/ mg Curcumin, a) 25/15, b) 25/25 c) 35/5 d) 35/15 e) 35/25. (B) Curcumin organogel rheogram, where a lineal viscoelastic region can be observed, also G" loss modulus and G' storage modulus can be observed. C) Nanogels formulated with different concentrations of curcumin, a) $1.5 \%$, b) $1 \%$ and c) $0.5 \%$ developed during the inverted tube test (D) Nanogel deformation sweep, the linear viscoelastic interval is indicated in the selected region.

Table 1 Logarithm of the storage module of the different concentrations of gelling agent in the organogels developed and shown in Fig.1 \% phospholipon 90H/mg curcumin, a) 25/15, b) $25 / 25$, c) $35 / 5$, d) $35 / 15$, e) $35 / 25$.

\begin{tabular}{cc}
\hline $\begin{array}{c}\text { \% Phospholipon } 90 \mathrm{H} / \\
\text { mg Curcumin }\end{array}$ & Ln G' $^{\prime}(\mathrm{Pa})$ \\
\hline $25 / 25$ & $13.8^{\mathrm{A}}$ \\
$25 / 15$ & $12.3^{\mathrm{B}}$ \\
$35 / 25$ & $11.2^{\mathrm{C}}$ \\
$35 / 15$ & $11.2^{\mathrm{C}}$ \\
$35 / 5$ & $9.0^{\mathrm{D}}$ \\
\hline
\end{tabular}

Entries that do not share the same letter are significantly different.

smaller Pa values (Fig. 1C); pH values were determined using a $\mathrm{pH}$ meter; all formulations had $\mathrm{pH}$ values ranging from 6.9 to 7.5 , close to that reported by Phatak and Chaudhari ${ }^{27)}$; these values are considered acceptable to avoid the risk of irritation after skin application. At a pH value closer to 6 , a gel using Carbopol $940^{\circledR}$ had a higher viscosity (lower Pa values) and bio-adhesiveness, most likely caused by a complete ionization of the polymeric main chain. This condition produces a repulsion between the native charges that in turn increases polymer swelling up to 1000 times its original volume, which results in stronger gels. These results are similar to those reported by
Chaudhary and co-workers ${ }^{28)}$, who developed a transdermal curcumin gel using Carbopol $940^{\circledR}$ with and without penetration enhancer, and performed in vitro permeation studies through skin cell lines. The above authors succeeded in developing the transdermal curcumin gels using Carbopol $940^{\circledR}$ as a release control polymer; these systems showed promising results.

\subsection{Rheological characterization}

Gel elasticity of curcumin NGs prepared by Carbopol 940 was examined. Then, gel elasticity (higher Pa values) increased with higher polymer concentration as shown in Table 2. Activation time was not considered for the Tukey analysis depicted in the table, because significant differences were observed in preliminary tests. This information concurs with the report by Islam and co-workers ${ }^{29)}$ who formulated gels by mixing Diclofenac ${ }^{\circledR}$ with curcumin. In Fig. 1D the loss modulus ( $G$ ") can be observed; this value represented less than $20 \%$ of the elastic modulus, indicating complete elasticity in the system. As deformation increased, elastic structures of the nanogels decreased abruptly. This behavior is consistent with another study ${ }^{29)}$ that performed a rheological characterization of gels containing Carbopol $940^{\circledR}$ as a crosslinker. As indicated by the author, smaller spheres lead to a smaller rupture tension; similarly, it could be observed, as Fig. 1 on this work indicates, before G' crossed with G", the loss modulus exhibited the characteristic waviness of viscoelastic systems.

Based on the results obtained in the development of the 
Table 2 Storage module for formulated CNGs formulated with different concentrations of carbopol $940^{\circledR}$ a) $1.5 \%$, b) $1 \%$, and c) $0.5 \%$ with 6 hours of activation time.

\begin{tabular}{|c|c|c|}
\hline \multicolumn{2}{|c|}{ Treatments } & \multirow{2}{*}{$\frac{\mathrm{G}^{\prime}(\mathrm{Pa})}{528.6^{\mathrm{A}}}$} \\
\hline \multirow{3}{*}{$1.5 \%$} & $24 \mathrm{~h}$ & \\
\hline & $12 \mathrm{~h}$ & $479.6^{\mathrm{B}}$ \\
\hline & $6 \mathrm{~h}$ & $518.6^{\mathrm{A}}$ \\
\hline \multirow{3}{*}{$1 \%$} & $24 \mathrm{~h}$ & $445.1^{\mathrm{C}}$ \\
\hline & $12 \mathrm{~h}$ & $477.0^{\mathrm{B}}$ \\
\hline & $6 \mathrm{~h}$ & $461.1^{\mathrm{BC}}$ \\
\hline \multirow{3}{*}{$0.5 \%$} & $24 \mathrm{~h}$ & $279.1^{\mathrm{E}}$ \\
\hline & $12 \mathrm{~h}$ & $257.5^{\mathrm{EF}}$ \\
\hline & $6 \mathrm{~h}$ & $339.9^{\mathrm{D}}$ \\
\hline
\end{tabular}

Entries that do not share the same letter are significantly different.

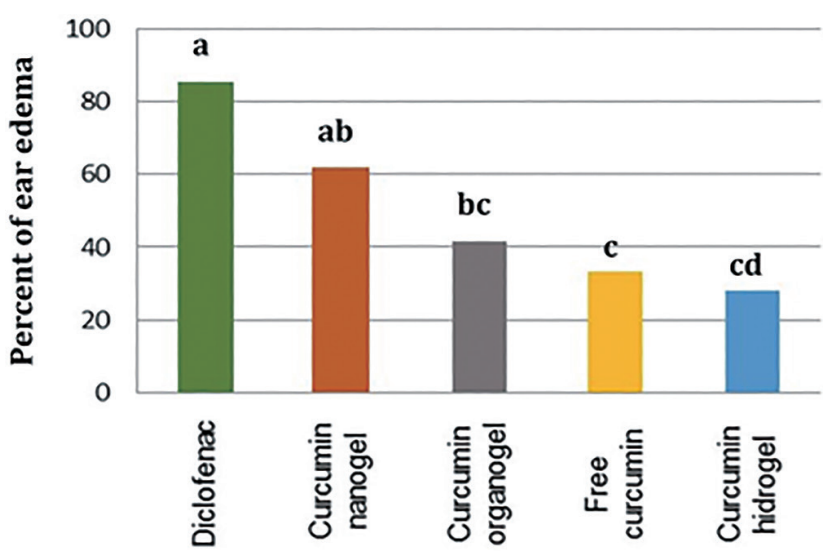

Fig. 2 Percentage of auricular edema inhibition from the treatments applied on the murine model. Bars that do not share a letter are significantly different.

CNGs and their rheological analysis, the CNG with the lowest concentration of Carbopol $940^{\circledR}$ was chosen among all the formulations because of the texture obtained once the system was neutralized, allowing a better application since it was less elastic. Higher concentrations of Carbopol $940^{\circledR}$ resulted in systems that were difficult to manipulate because of their hardness (higher Pa values); also, as there were no significant differences with respect to time, the CNG with the shortest activation time was employed for the in vivo assessment. The final Carbopol $940^{\circledR}$ concentration used for the system that was applied to the mice was in agreement with that used in a previous study where vitamin A palmitate-carrying hydrogels (unlike the CNG hydrogels have no nanometric particles, and contain only water, carbopol and curcumin)were developed, obtaining viscous systems ${ }^{30)}$.
Table 3 Weight (mg) and Tukey analysis of dissected mouse ears, to which treatments were applied.

\begin{tabular}{lc}
\hline \multicolumn{1}{c}{ Group } & Weight $(\mathrm{mg})$ \\
\hline Healthy control & $9.90 \pm 0.7^{\mathrm{a}}$ \\
2-(2,6-dichloranilino) phenylacetic acid & $11.2 \pm 0.8^{\mathrm{ab}}$ \\
Nanogel (CNGs) & $13.3 \pm 0.6^{\mathrm{bc}}$ \\
Organogel (COGs) & $14.7 \pm 0.8^{\mathrm{cd}}$ \\
Free Curcumin (FC) & $15.3 \pm 0.6^{\mathrm{d}}$ \\
Hydrogel (CHGs) & $16.3 \pm 0.8^{\mathrm{de}}$ \\
Edema control & $18.9 \pm 0.6^{\mathrm{e}}$ \\
\hline
\end{tabular}

Means that do not share the same letter are significantly different.

\subsection{Bioactivity of curcumin NG}

To assess the effects of the various treatments (CNGs, COGs, Diclofenac ${ }^{\circledR}$, CHGs and FC) on atrial edema; this condition was induced by multiple applications of TPA to produce a localized inflammation in the ear. The anti-inflammatory response was determined by the degree of swelling of the treated ears compared to ears with no treatment ${ }^{31}$. The results are expressed as the weight of the ear and as a percentage of inhibition, in contrast to the control group that did not receive any treatment. Comparison of the weight means of the ears from different treatments was carried out (Fig. 2). Healthy mice that were only given Diclofenac ${ }^{\circledR}$ had the smallest means, and showed the smallest edema. On the other hand, mice treated with the CNGs were the only subjects that did not have significant differences to the reference drug. In the case of the COGs and $\mathrm{FC}$, the response of the mean values was in an intermediate scale, resulting in statistical similarity. However, mice treated with the CHGs had the highest mean value of the treatments, and were no significantly different to mice treated only with TPA. When analyzing the percent inhibition of stablished treatments, we observed that CNGs had a higher anti-inflammatory activity $(61.8 \%)$ than COGs (45.9\%), FC (33.2\%) and CHGs (28.1\%). Diclofenac ${ }^{\circledR}$ accomplished the highest inhibition $(85.4 \%)$; however, it caused the death of $2(40 \%)$ of the animals. The lethal effect of Diclofenac ${ }^{\circledR}$ has been associated with up to $40 \%$ of increased probability of cardiovascular risks, as it has been reported in several studies ${ }^{32,33)}$.

Also, it is highly recommended to use the drug only for short periods of time and, if possible, avoid it. Table 3 shows the weights and percent inhibition of the different treatments. The results obtained are comparable to a study conducted by Sun and co-workers ${ }^{34)}$ who investigated the effect of a transdermal curcumin gel in a model of atrial edema induced by TPA in K14-VEGF transgenic mice. The increase in bone thickness was employed to indicate the extent of inflammation. As in our study, on days 2-4 after 
the start of treatment with TPA, ear skin started to display signs of thickening and erythema development with increased severity that peaked on days 6-10.

H\&E staining analysis results from ear skin samples treated with TPA showed thickened subcutaneous tissue and mild epidermal enlargement compared to the control group (Fig. 3A, B). The increase in thickness was attributed to the rise in the number of inflammatory cells in the subcutaneous layer. In another study ${ }^{35)}$, the anti-inflammatory activity of curcumin was evaluated in vitro using a septic shock model induced by LPS. Exosomes (30-100 nm nanoparticles secreted by cells in the extracellular environment) formed a complex with curcumin. Exosomal curcumin potentiates apoptosis in CD11b + Gr-1 + cells and regulates the activity in several crucial transcription factors in inflammation, including NF-кB, STAT3 and Nrf2; therefore, additional biological effects on cell proliferation, apoptosis, induction of cytokines and an antioxidant effect can also contribute to the prevention of induced septic shock ${ }^{35)}$.

\subsection{Histopathological analysis}

To verify our data, histological sections of the ears were prepared and examined using an optical microscope. The group treated with TPA showed a chronic and acute inflammation with moderate ulceration, i.e., loss of continuity of the epithelium by chemical effects, clear evidence of epidermal hyperplasia, edema, and dermal inflammatory cell infiltration along with possible disruption of connective tissue (Fig. 3A, B); these observations concur with those of Paul \& $\mathrm{Kang}^{36}$. It is possible to notice a disarrangement in cellular structures with the presence of multinucleated cells through microscopical observation ${ }^{37)}$. Ear portions obtained from the group treated with Diclofenac ${ }^{\circledast}$ (Fig. 3C, D) showed similar cellular structure to the healthy control group. Despite the fact that better results were observed compared to the other treatments, and as mentioned above, $40 \%$ of the animals of this group died before the end of the experiment. Their demise could have been caused by the adverse effects of the drug, as previously mentioned. In a meta-analysis work it was concluded that Diclofenac ${ }^{\circledR}$ increased the risk of cardiovascular complications when compared with a placebo. In view of well-established damage and duration of treatment, intermittent, short-term use, a dose of less than $75 \mathrm{mg} /$ day was recommended. Diclofenac ${ }^{\circledR}$ has been associated with a statistical-
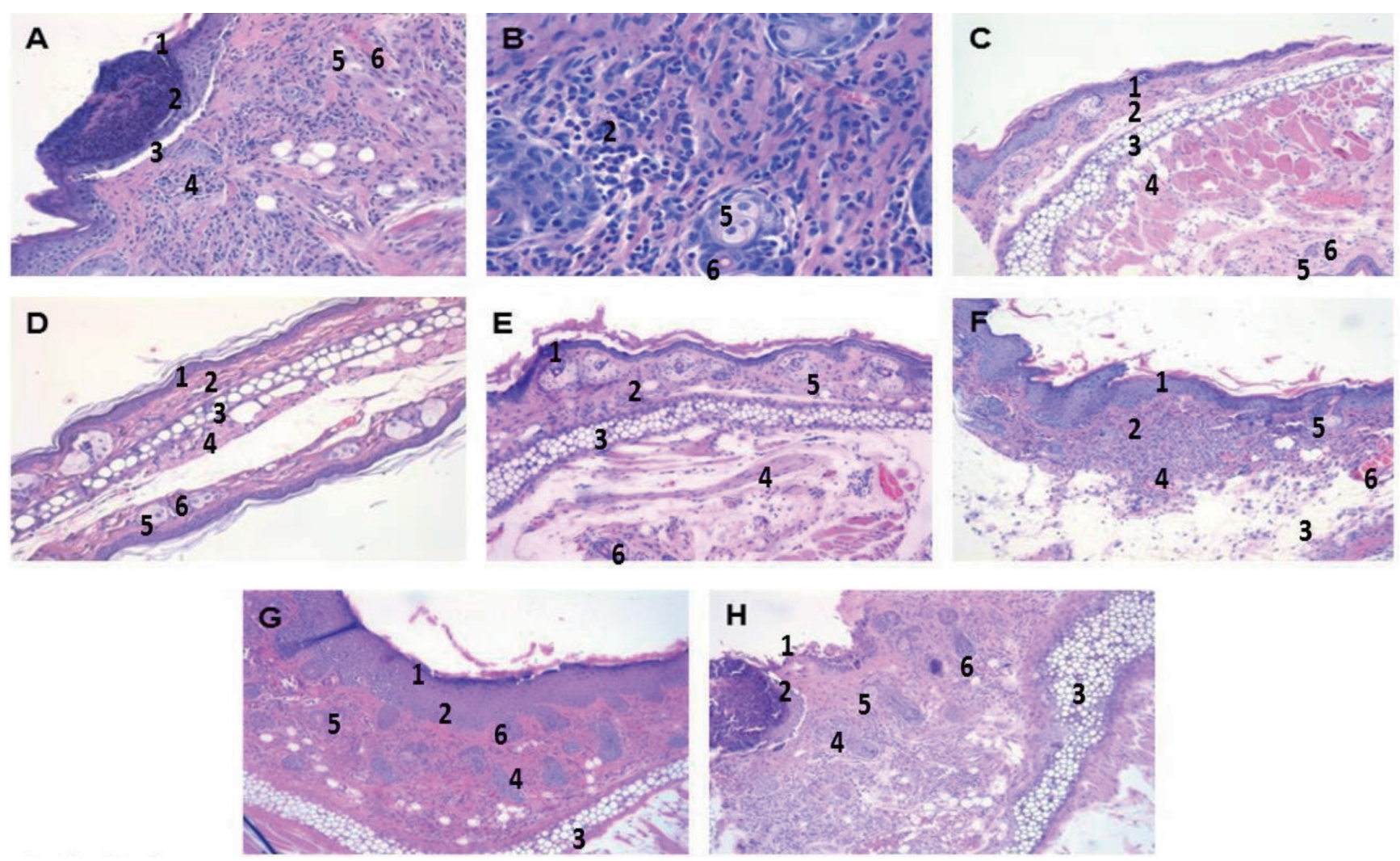

Fig. 3 Histological analysis of the mouse ear stained with H\&E: (A,B) Sick control group at 10x and 40x; (C,D) Healthy control group at 10x and 40x; (E) Nanogel treated group at 10x; (F) Organogels treated group at 10x; (G) Free curcumin treated group at 10x; (H) Hydrogel treated group at 10x. Epidermis (1), Dermis (2), Elastic cartilage (3), Striated muscle (4), Sebaceous glands (5), Hair follicles (6). 
ly significant $22 \%$ increase in risk at low doses ${ }^{38)}$. The group treated with the CNGs displayed the best results out of the proposed treatments, with only an incipient acute inflammation in the dermis.

This result may be attributed to the CNGs ability to increase curcumin release and the data is similar to that of Al-Rohaimi ${ }^{39)}$, who evaluated the anti-inflammatory effects of curcumin in a nanogel, and compared with a Diclofenac ${ }^{\circledast}$ gel in a plantar edema induced by carrageenan in rats. After its application, the inhibition \% of the nanogel did not show significant difference with the commercial gel $(78.54 \%$ and $81.13 \%$, respectively). In this study it was also proven that curcumin had permeability problems if particles had a larger size; so, the nanogel increased permeability and effectiveness of the bioactive compound. The diagnosis proposed that this group had a mild chronic inflammation (Fig. 3E). Although the inhibition was $61 \%$, it was smaller than values reported by Wang and co-work$\mathrm{ers}^{40)}$, in which curcumin was administered through a nanoemulsion and its effect was tested on atrial edema induced by TPA, and obtained an $85 \%$ decrease in ear weight.

The COGs produced a slight loss of inflammation; when analyzing the histological section, severe chronic and acute inflammation could still be observed (Fig. 3F), with a clear increase in cellularity in the stroma, which was secondary to the presence of an inflammatory infiltrate and foci, thus increasing the sub-epithelial inflammatory infiltrate. The $45 \%$ inhibition of auricular edema produced in this study contrasts with the results obtained by Esposito and coworkers ${ }^{41)}$, who compared the preventive effect of applying a curcumin organogel and an aqueous dispersion of monoolein on skin to be later exposed to UV radiation and measured the degree of erythema. The results showed that the organogel inhibited $50 \%$ of skin damage in comparison to $10 \%$ by the aqueous monoolein dispersion.

Huang and co-workers ${ }^{42)}$, demonstrated that the application of $1 \mu \mathrm{L}$ of curcumin before a single application of TPA inhibited the induction of edema by $99 \%$. Additionally, Yadav and co-workers ${ }^{43}$ applied topical curcumin, which significantly inhibited the inflammation induced by TPA, and resulted in reductions in ear redness. Ears treated with FC had a decline in atrial edema, which exhibited significant differences compared to the group with edema. However, acute and chronic inflammation was also observed, as well as micro-abscesses, as shown in Fig. 3G.

Finally, the group treated with the CHGs displayed severe inflammation, as well as abscesses that could be distinguished in the epidermis and corneal layer. An acute predominant inflammatory infiltrate was identified in the thickness of the dermis, which extended to the muscular layer(Fig. 3H). This group did not show significant differences with the group treated only with TPA, because the system showed a high viscosity and did not manage to remain in the damaged site or achieve the desired effects. This result is lower than that reported by Al-Rohaimi ${ }^{39)}$, who used a hydrogel in a plantar edema induced by carrageenan and achieved an inflammation inhibition of $40 \%$; however, this work was preventive, whereas our multiple application model of TPA was focused as curative.

\section{Conclusions}

Successful formulation of organogels and nanogels was achieved and used as vehicles to carry curcumin for topical application on a model mouse ear edema. Thermograms displayed a linear growth in enthalpy with respect to the concentration of the gelling agent used in COGs. By means of the deformation sweeps, both viscous and elastic behavior were confirmed in both systems. CNGs had a greater anti-inflammatory activity (61.8\%) than the COGs (45.9\%), FC $(33.2 \%)$ and CHGs $(28.1 \%)$. Diclofenac ${ }^{\circledR}$ achieved the highest edema inhibition ( $85.4 \%$ ); however, $40 \%$ of the study subjects in this group died from exposure to the drug.

\section{Acknowledgments}

The authors gratefully appreciate the financial support of the National Council for Science and Technology of México (CONACYT), through the grant 250784, and the graduate studies scholarship of author Gonzalez-Ortega.

\section{References}

1) Leonardi, G.C.; Accardi, G.; Monastero, R.; Nicoletti, F.; Libra, M. Ageing: from inflammation to cancer. Immun. Ageing 15, 1(2018).

2) Azmir, J.; Zaidul, I.S.M.; Rahman, M.M.; Sharif, K.M.; Mohamed, A.; Sahena, F.; Omar, A.K.M. Techniques for extraction of bioactive compounds from plant materials: A review. J. Food Eng. 117, 426-436 (2013).

3) Awika, J.M.; Duodu, K.G. Bioactive polyphenols and peptides in cowpea (Vigna unguiculata) and their health promoting properties: a review. J. Funct. Foods 38, 686-697 (2017).

4) García-Varela, R.; Ramírez, O.R.F.; Serna-Saldivar, S.O.; Altamirano, J.; Cardineau, G.A. Cancer cell specific cytotoxic effect of Rhoeo discolor extracts and solvent fractions. J. Ethnopharmacol. 190, 46-58(2016).

5) Chen, S.; Li, Y.; Chen, S.; Tan, J.; Ou, T.; Huang, Z. Design, synthesis, and biological evaluation of curcumin analogues as multifunctional agents for the treatment of Alzheimer's disease. Bioorg. Med. Chem. 19, 5596$5604(2011)$. 
6) Shanmugam, M.; Rane, G.; Kanchi, M.; Arfuso, F.; Chinnathambi, A.; Zayed, M.; Sethi, G. The multifaceted role of curcumin in cancer prevention and treatment. Molecules 20, 2728-2769 (2015).

7) Tiwari, B.; Pahuja, R.; Kumar, P.; Rath, K. Nanotized curcumin and miltefosine, a potential combination for treatment of experimental visceral Leishmaniasis. Antimicrob. Agents Chemother. 61, 1-13(2017).

8) Yarla, N.S.; Bishayee, A.; Sethi, G.; Reddanna, P.; Kalle, A.M.; Dhananjaya, B.L.; Dowluru, K.S.; Chintala, R.; Duddukuri, G.R. Targeting arachidonic acid pathway by natural products for cancer prevention and therapy. Semin. Cancer Biol. 40-41, 48-81 (2016).

9) Ni, H.; Jin, W.; Zhu, T.; Wang, J.; Yuan, B.; Jiang, J.; Liang, W.; Ma, Z. Curcumin modulates TLR4/NF-кB inflammatory signaling pathway following traumatic spinal cord injury in rats. J. Spinal Cord Med. 38, 199206 (2015).

10) Yuan, J.; Liu, R.; Ma, Y.; Zhang, Z.; Xie, Z. Curcumin attenuates airway inflammation and airway remolding by inhibiting NF- $\mathrm{KB}$ signaling and COX-2 in cigarette smoke-induced COPD Mice. Inflammation 41, 18041814 (2018).

11) Duvoix, A.; Blasius, R.; Delhalle, S.; Schnekenburger, M.; Morceau, F.; Henry, E.; Diederich, M. Chemopreventive and therapeutic effects of curcumin. Cancer Lett. 223, 181-190 (2005).

12) Sordillo, P.; Helson, L.; Pharma, S. Curcumin and cancer stem cells : Curcumin has asymmetrical effects on cancer and normal stem cells. Anticancer Res. 614, 599-614 (2015).

13) Joe, B.; Vijaykumar, M.; Lokesh, B. Biological properties of curcumin-cellular and molecular mechanisms of action. Crit. Rev. Food Sci. Nutri. 44, 97-111(2004).

14) Nazari-Vanani, R.; Moezi, L.; Heli, H. In vivo evaluation of a self-nanoemulsifying drug delivery system for curcumin. Biomed. Pharmacother. 88, 715-720 (2017).

15) Naksuriya, O.; Okonogi, S.; Schiffelers, R.; Hennink, W. Curcumin nanoformulations: A review of pharmaceutical properties and preclinical studies and clinical data related to cancer treatment. Biomaterials 35, 33653383 (2014).

16) Tuttle, S.; Hertan, L.; Katz, J.S. Indian gold treating cancer in the age of nano. Cancer Biol. Ther. 11, 474476 (2014).

17) Gholibegloo, E.; Mortezazadeh, T.; Salehian, F.; Ramazani, A.; Amanlou, M.; Khoobi, M. Improved curcumin loading, release, solubility and toxicity by tuning the molar ratio of cross-linker to $\beta$-cyclodextrin. Carbohydr. Polym. 213, 70-78(2019).

18) Dassanayake, L.S.K.; Kodali, D.K.; Ueno, S.; Sato, K. Physical properties of rice bran wax in bulk and organogels. J. Am. Oil Chem. Soc. 86, 1163-1173
(2009).

19) Raj, R.; Mongia, P.; Ram, A.; Jain, N.K. Enhanced skin delivery of aceclofenac via hydrogel-based solid lipid nanoparticles. Artif. Cells Nanomed. Biotechnol. 44, 1434-1439 (2016).

20) Panonnummal, R.; Jayakumar, R.; Sabitha, M. Comparative anti-psoriatic efficacy studies of clobetasol loaded chitin nanogel and marketed cream. Eur. J. Pharm. Sci. 96, 193-206 (2016).

21) Ahad, A.; Al-Saleh, A.A.; Al-Mohizea, A.M.; Al-Jenoobi, F.I.; Raish, M.; Yassin, A.E.B.; Alam, M.A. Formulation and characterization of Phospholipon $90 \mathrm{G}$ and tween 80 based transfersomes for transdermal delivery of eprosartan mesylate. Pharm. Dev. Technol. 23, 787$793(2017)$.

22) Nagahama, K.; Kimura, Y.; Takemoto, A. Living functional hydrogels generated by bioorthogonal crosslinking reactions of azide-modified cells with alkynemodified polymers. Nat. Commun. 9, 2195 (2018).

23) Shi, R.; Zhang, Q.; Vriesekoop, F.; Yuan, Q.; Liang, H. Preparation of organogel with tea polyphenols complex for enhancing the antioxidation properties of edible oil. J. Agric. Food Chem. 62, 8379-8384(2014).

24) Karimineghlani, P.; Emmons, E.; Green, M.J.; Shamberger, P.; Sukhishvili, S.A. A temperature-responsive poly (vinyl alcohol) gel for controlling fluidity of an inorganic phase change material. J. Mater. Chem. A 5, 12474-12482 (2017).

25) Stanley, P.L.; Steiner, S.; Havens, M.; Tramposch, K.M. Mouse skin inflammation induce by multiple topical application of 12-OTetradecanoylphorbol-13-acetate. Skin Pharmacol. 4, 262-271 (1991).

26) Bhatia, A.; Singh, B.; Raza, K.; Wadhwa, S.; Katare, O.P. Tamoxifen-loaded lecithin organogel(LO) for topical application: Development, optimization and characterization. Int. J. Pharm. 444, 47-59 (2013).

27) Phatak, A.A.; Chaudhari, P.D. Development and evaluation of nanogel as a carrier for transdermal delivery of Aceclofenac. Asian J. Pharm. Tech. 2, 125-132 (2012).

28) Chaudhary, H.; Kohli, K.; Amin, S.; Rathee, P.; Kumar, V. Optimization and formulation design of gels of Diclofenac and curcumin for transdermal drug delivery by Box-Behnken statistical design. J. Pharm. Sci. 100, 580-593 (2011).

29) Islam, M.T.; Ciotti, S.; Ackermann, C. Rheological characterization of topical carbomer gels neutralized to different pH. Pharm. Res. 21, 1192-1199 (2004).

30) Oliveira, J.S.; Aguiar, T.A.; Mezadri, H.; Henrique dos Santos, O.D. Attainment of hydrogel-thickened nanoemulsions with tea tree oil(Melaleuca alternifolia) and retinyl palmitate. Afr. J. Biotechnol. 10, 1301413018 (2011).

31) Shah, P.P.; Desai, P.R.; Patel, A.R.; Singh, M.S. Skin 
permeating nanogel for the cutaneous co-delivery of two anti-inflammatory drugs. Biomaterials 33, 16071617 (2012).

32) Mcgettigan, P.; Henry, D. Cardiovascular risk and inhibition of cyclooxygenase. J. Am. Med. Assoc. 296, 1633-1644(2006).

33) Trelle, S.; Reichenbach, S.; Wandel, S.; Hildebrand, P.; Tschannen, B.; Villiger, P.M.; Egge, M.; Jüni, P. Cardiovascular safety of non-steroidal anti-inflammatory drugs: network meta-analysis. Br. Med. J. 342, c7086 (2011).

34) Sun, J.; Zhao, Y.; Jin, H.; Hu, J. Curcumin relieves TPAinduced Th1 inflammation in K14-VEGF transgenic mice. Int. Immunopharmacol. 25, 235-241 (2015).

35) Sun, D.; Zhuang, X.; Xiang, X.; Liu, Y.; Zhang, S.; Liu, C.; Zhang, H. A novel nanoparticle drug delivery system: The anti-inflammatory activity of curcumin is enhanced when encapsulated in exosomes. Mol. Ther. 18, 1606-1614 (2010).

36) Paul, S.; Kang, S.C. Natural polyamine inhibits mouse skin inflammation and macrophage activation. Inflamm. Res. 62, 681-688(2013).

37) Meng, H.; Liu, Y.; Lai, L. Diverse ways of perturbing the human arachidonic acid metabolic network to control inflammation. Accounts Chem. Res. 48, 22422250 (2015).
38) Mcgettigan, P.; Henry, D. Use of non-steroidal anti-inflammatory drugs that elevate cardiovascular risk : An examination of sales and essential medicines lists in low-, middle-, and high- income countries. PLoS Med. 10, e1001388 (2013).

39) Al-Rohaimi, A.H. Comparative anti-inflammatory potential of crystalline and amorphous nano curcumin in topical drug delivery. J. Oleo Sci. 40, 27-40 (2015).

40) Wang, X.; Jiang, Y.; Wang, Y.W.; Huang, M.T.; Ho, C.T.; Huang, Q. Enhancing anti-inflammation activity of curcumin through O/W nanoemulsions. Food Chem. 108, 419-424 (2008).

41) Esposito, E.; Ravani, L.; Mariani, P.; Huang, N.; Boldrini, P.; Drechsler, M.; Puglia, C. Effect of nanostructured lipid vehicles on percutaneous absorption of curcumin. Eur. J. Pharm. Biopharm. 86, 121-132 (2012).

42) Huang, M.-T.; Robertson, F.M.; Lysz, T.; Ferraro, T.; Wang, Z.Y.; Georgiadis, C.A.; Conney, A.H. Inhibitory effects of curcumin on in vitro lox and cox activities in mouse epidermis. Cancer Res. 507, 338-349 (1992) .

43) Yadav, K.S.; Maan, P.; Bhatia, S.; Yadav, N.P. Exploration of anti-inflammatory activity of turmeric and onion combination on phorbol ester induced ear inflammation in mice. Ann. Phytomed. 3, 50-54(2014). 